\title{
Fluid modeling of a microwave micro-plasma at atmospheric pressure
}

\author{
J. Gregório ${ }^{1,2}$, C. Boisse-Laporte ${ }^{2}$, and L.L. Alves ${ }^{1, a}$ \\ 1 Instituto de Plasmas e Fusão Nuclear - Laboratório Associado, Instituto Superior Técnico, 1049-001 Lisboa, Portugal \\ 2 Laboratoire de Physique des Gaz et des Plasmas, Université Paris-Sud/CNRS, 91405 Orsay, France
}

Received: 28 February 2010 / Accepted: 5 March 2010

Published online: 31 March 2010 - (c) EDP Sciences

Abstract. This is an erratum to Eur. Phys. J. Appl. Phys. 49, 13102 (2010), DOI: 10.1051/epjap/2009180.

An error occurred in the presentation of the following equations.

Equation (7) and its description, on page 13102-p2 of the original article, should read

$$
\frac{1}{2} n_{e} \mu_{e}\left|\widetilde{E}_{\mathrm{hf}}\right|^{2}=\Gamma_{e} E_{\mathrm{dc}}+\frac{d \Gamma_{\varepsilon}}{d y}+n_{e} \Theta_{\mathrm{coll}}+n_{e} \sum_{r=5}^{7} \Theta_{\mathrm{kin}, r}
$$

$$
[\ldots]
$$

Here, $\Theta_{\text {coll }}$ is the power lost per electron due to elastic, inelastic, and superelastic electron-neutral collisions (thus, including also reactions $1-3$ in Tab. 1); $\Theta_{\mathrm{kin}, r}$ is the power lost per electron due to all other collisions between electrons and heavy particles (reactions $5-7$ in Tab. 1); [... the terms on the right-hand side of equation (7) represent $[\ldots]$; and (iii) the power lost in collisions between electrons and heavy particles (for the last two terms).
Equation (9) and its description, on pages 13102-p2 and 13102-p3 of the original article, should read

$$
\begin{aligned}
-\frac{d}{d y}\left(\lambda_{T} \frac{d T_{g}}{d y}\right)= & n_{e} \Theta_{\mathrm{el}}+\left(\sum_{p} \Gamma_{p}\right) E_{\mathrm{dc}} \\
& +n_{e} \sum_{r=5}^{7} \Theta_{\mathrm{kin}, r}-\sum_{h} S_{\mathrm{kin}, h} \varepsilon_{h} .
\end{aligned}
$$

Here, [...] $S_{\text {kin }, h}$ is the net production rate of heavy species $h=\mathrm{Ar}^{+}, \operatorname{Ar}_{2}^{+}, \operatorname{Ar}(4 \mathrm{~s})$, with excitation energy $\varepsilon_{h}$, due to the kinetic reactions $4-8$ (see Tab. 1). [...] the terms on the right-hand side of equation (9) represent [...] (iii) the energy transferred from electrons to the gas via the kinetic reactions 5-7 (see Tab. 1); and (iv) the energy transferred to the gas potential energy, after production of heavy species $h$ via the kinetic reactions $4-8$ (see Tab. 1).

The authors apologise for these errors.

\footnotetext{
${ }^{a}$ e-mail: llalves@ist.utl.pt
} 